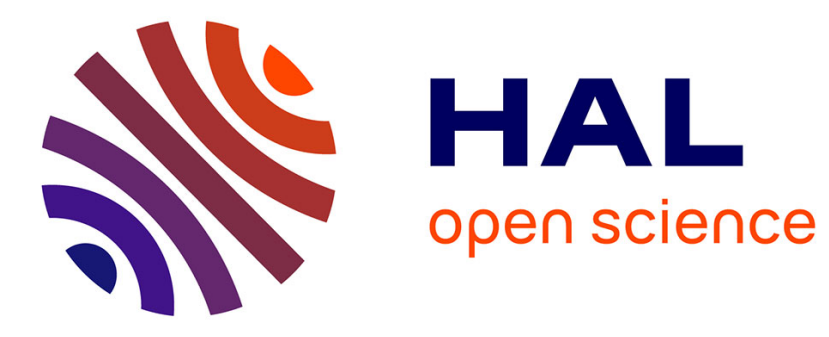

\title{
Calibration Challenges for the Biomass P-Band SAR Instrument
}

Shaun Quegan, Mark R. Lomas, Konstantinos Panagiotis Papathanassiou, Junsu S. Kim, Stefano Tebaldini, Davide Giudici, Michele Scagliola, Pietro Guccione, Jørgen Dall, Pascale Dubois-Fenandez, et al.

\section{To cite this version:}

Shaun Quegan, Mark R. Lomas, Konstantinos Panagiotis Papathanassiou, Junsu S. Kim, Stefano Tebaldini, et al.. Calibration Challenges for the Biomass P-Band SAR Instrument. 38th Annual IEEE International Geoscience and Remote Sensing Symposium, IGARSS 2018, Jul 2018, VALENCIA, Spain. 10.1109/IGARSS.2018.8518646 . hal-02908484

\section{HAL Id: hal-02908484 \\ https://hal.science/hal-02908484}

Submitted on 8 Nov 2021

HAL is a multi-disciplinary open access archive for the deposit and dissemination of scientific research documents, whether they are published or not. The documents may come from teaching and research institutions in France or abroad, or from public or private research centers.
L'archive ouverte pluridisciplinaire HAL, est destinée au dépôt et à la diffusion de documents scientifiques de niveau recherche, publiés ou non, émanant des établissements d'enseignement et de recherche français ou étrangers, des laboratoires publics ou privés.

\section{(c)(1)}

Distributed under a Creative Commons Attribution| 4.0 International License 


\title{
CALIBRATION CHALLENGES FOR THE BIOMASS P-BAND SAR INSTRUMENT
}

\author{
S. Quegan ${ }^{1}$, M. Lomas ${ }^{1}$, K. P. Papathanassiou ${ }^{2}$, J-S. Kim ${ }^{2}$, S. Tebaldini ${ }^{3}$, D. Giudici ${ }^{4}$, M. Scagliola ${ }^{4}$, P. \\ Guccione $^{8}$, J. Dall ${ }^{5}$ P. Dubois-Fernandez ${ }^{6}$ and P. Paillou ${ }^{7}$ \\ ${ }^{1}$ University of Sheffield, UK and UK National Centre for Earth Observation; ${ }^{2}$ DLR, Munich, Germany; \\ ${ }^{3}$ Politecnico di Milano, Italy; ${ }^{4}$ Aresys, Milano, Italy; ${ }^{5}$ Technical University of Denmark; ${ }^{6}$ ONERA, \\ France; ${ }^{7}$ University of Bordeaux, France; ${ }^{8}$ Politecnico di Bari, Italy
}

\begin{abstract}
The BIOMASS mission gives completely new challenges in external calibration arising from the orbital pattern needed for the tomographic and Pol-InSAR phases of the mission, the strong effects of the ionosphere at P-band, and the lack of pre-existing P-band data except over very limited parts of the globe. Together these create problems that can only be solved by combining infrequent visits to instrumented calibration sites with systematic exploitation of the properties of distributed targets and targets of opportunity. Proposed approaches to performing radiometric and polarimetric calibration are described, together with meeting geolocation accuracy requirements.
\end{abstract}

Index Terms - Radiometry, polarimetry, geolocation, ionospheric correction, calibration

\section{INTRODUCTION}

BIOMASS will be the first P-band mission in space, the first systematic use of Polarimetric Interferometry (Pol-InSAR) for forest height measurement, and the first radar tomographic mission. Its primary aim is to acquire global forest biomass and height outside the region covered by Space Object Tracking Radar (SOTR) restrictions (this includes the N American continent, Europe and the Arctic), but secondary objectives include coverage of the desert areas and Antarctica. Achieving mission objectives needs well-calibrated measurements for which both system and ionospheric effects have been compensated. This raises many new challenges, particularly with regard to radiometry, polarimetry and geolocation, with fewer issues for PolInSAR and tomography. These were explored in the BIOMASS External Calibration Study [1] on which this paper is based. The BIOMASS calibration strategy is strongly affected by the orbital pattern and the ionosphere (Sections 2 \& 3). Radiometric calibration, including antenna pattern and pointing error compensation is discussed in Section 4, while polarimetric calibration and geolocation issues are discussed in Sections 5 and 6.

\section{THE BIOMASS ORBITAL PATTERN}

BIOMASS will have an orbital pattern organized into three phases. The 3-month Commissioning Phase (CP) is designed to allow 21 passes over a transponder at different incidence angles in order to measure the 2-D antenna pattern and test the polarimetric calibration. The following Tomographic Phase will provide tomographic mapping of all forest areas outside the SOTR zone. This requires 425 days ( 14 months) in order to provide 7 passes, each separated by 3 days, to be used for tomography over all continental areas outside the SOTR zone.. The remainder of the 5-year mission will be taken up by the Interferometric Phase, during which each cycle will require 219 days ( $\sim 7$ months) to provide global coverage using 3-baseline Pol-InSAR with 3-day repeat. This orbit pattern has major implications for BIOMASS calibration, since the transponder is only visited at infrequent intervals after the $\mathrm{CP}$, and even then only at a limited set of incidence angles. Hence routine calibration needs to exploit targets of opportunity, including distributed targets and Permanent Point Scatterers (PPSs) (Section 4). Candidate targets of opportunity will be characterized during the $\mathrm{CP}$.

\section{IONOSPHERIC EFFECTS}

The ionosphere affects all measurements made by BIOMASS, the main effects being Faraday rotation (FR), range delay and, at high latitudes, scintillation (the BIOMASS dawn-dusk orbit is specifically chosen to avoid the severe ionospheric disturbances in the post-sunset equatorial ionosphere). FR in itself not a problem since it is readily compensated. The measured polarimetric scattering matrix, M, can be written, in the absence of system errors, as

$$
\mathbf{M}=\mathbf{F S F}
$$

where $\mathbf{S}$ is the true scattering matrix and

$$
\mathbf{F}=\left[\begin{array}{cc}
\cos \Omega & \sin \Omega \\
-\sin \Omega & \cos \Omega
\end{array}\right] \text {. }
$$

where $\Omega$ is the one-way FR angle given by

$$
\Omega=\frac{A}{f^{2}} \times \mathbf{B} \cdot \mathbf{k} \times \text { TEC. }
$$


Here TEC is the Total Electron Content, $\mathbf{B}$ is the magnetic field, $\mathbf{k}$ is the unit propagation vector, $f$ is the wave frequency, and $A=\frac{c}{2 \pi} \frac{e}{m_{e}} r_{e}$, where $c$ is the speed of light, $e$ and $m_{e}$ are the electron charge and mass, respectively, and $r_{e}$ is the classical electron radius. Hence $\mathbf{S}$ can be estimated as

$$
\hat{\mathbf{S}}=\hat{\mathbf{F}}^{-1} \mathbf{M} \hat{\mathbf{F}}^{-1}
$$

once we have an estimate of $\Omega$. The well-known Bickel and Bates algorithm has been shown to be the best estimator of $\Omega[2]$ and has variance

$$
\operatorname{var}(\hat{\Omega})=\frac{1}{16} \frac{1-\gamma^{2}}{2 \gamma L}
$$

where $L$ is the number of independent samples in the estimation window,

$$
\gamma=\frac{1}{1+\frac{\sigma_{n}}{\left\langle\left|S_{h h}+S_{v v}\right|^{2}\right\rangle}}=\frac{1}{1+\mathrm{SNR}^{-1}}
$$

and $\sigma_{n}=\sigma_{n h h}+\sigma_{n h v}+\sigma_{n v h}+\sigma_{n v v}$ is the sum of the noise powers in the 4 polarimetric channels.

Measurements of FR are also crucial in estimating and compensating ionospheric effects using the relations between one-way phase delay, $\phi_{d}$, two-way slant range delay, $r_{s d}$, and TEC:

$$
\begin{aligned}
\phi_{d} & =\frac{c r_{e}}{f} \mathrm{TEC} \\
r_{s d} & =\frac{c^{2} r_{e}}{2 \pi} \frac{1}{f^{2}} \mathrm{TEC}
\end{aligned}
$$

Using (3) then gives a simple frequency-independent relation between slant range delay and $\Omega$ :

$$
\frac{\Omega}{r_{s d}}=\frac{1}{c} \frac{e}{m_{e}} \mathbf{B} \cdot \mathbf{k}=586.3 \times \mathbf{B} \cdot \mathbf{k}
$$

\section{RADIOMETRIC CALIBRATION}

For a point target with scattering matrix $\left[S_{p q}\right]$, where $p$ and $q$ denote polarization, the measured voltage $V_{p q}$ is related to the desired quantity $S_{p q}$ at position $(x, y)$ by the idealized relation

$$
V_{p q}=\sqrt{K_{s}} e^{i \phi_{s}} S_{p q}(x, y) \otimes h(x, y)+\sqrt{K_{n}} n(x, y)
$$

where $h$ is the spatially variant 2-D point spread function and $\otimes$ denotes 2-D convolution. $K_{s}$ and $\phi_{s}$ represent the gain and phase imposed on $S_{p q}$ by the overall radar system, $n$ is additive noise, and $K_{n}$ is the radar gain for the noise; all four of these quantities may be spatially dependent.

In simplified form

$$
K_{s}=\alpha G_{A}^{2}\left(\theta_{e l}, \theta_{a z}\right) G_{E} G_{P}(x, y)
$$

where $\alpha$ contains the range and frequency dependence, $G_{A}$ is the antenna pattern as a function of elevation and azimuth angles $\left(\theta_{e l}, \theta_{a z}\right), G_{E}$ is the electronic receiver gain (incorporating system and atmospheric losses) and $G_{P}$ is the processor gain.

Complete calibration of the complex SAR image requires determination and correction for $K_{s}$ and $\phi_{s}$ in the presence of the noise contribution. For purely radiometric calibration, this requires correction of spatial variations imposed by the antenna pattern, together with absolute calibration against reference targets.

\subsection{Antenna pattern and pointing corrections}

Because the antenna is deployable and too large to be characterized on ground, the first part of the Commissioning Phase (CP) will be used to characterize the antenna pattern and calibrate the system pointing using measurements from one or more transponders in recorder mode. These measurements will be used in an antenna model to characterize the actual antenna pattern and pointing.

After the $\mathrm{CP}$, revisits to the transponder(s) are infrequent and with a very limited range of elevation angles provided by the 3 (7) acquisitions during the Interferometric (Tomographic) Phases. Targets of opportunity (PPSs and distributed targets) may be used to fill the gaps in time and incidence angle if the BIOMASS antenna and its radiometry are well characterized during the first part of the CP. The BIOMASS data themselves can then be used in the second part of the $\mathrm{CP}$ to characterize the radiometric response of selected targets of opportunity. These targets can then be used routinely during the rest of the mission to monitor the elevation pointing by cross-correlating the observed and expected elevation antenna patterns. Continual monitoring of the azimuth pointing can be determined in-flight by exploiting the Doppler Centroid characteristics of acquisitions over homogeneous distributed natural targets.

\subsection{Absolute Radiometry}

Absolute radiometric calibration requires reference targets with known RCS (point targets) or $\sigma^{0}$ (distributed targets). These must be temporally stable and have large Signal to Clutter Ratio (point targets) or large Signal to Noise Ratio (SNR; distributed targets). We further require that distributed targets are spatially homogeneous at scales large enough for many measurements with the same radiometric properties to be made.

For many previous missions (e.g. Envisat) the Amazon rainforest could be treated as a stable distributed target and used for monitoring purposes, but BIOMASS is designed to measure variation and change in such forests. As a result, other targets of opportunity are needed, of which there appear to be three potential types: deserts, icesheets \& PPSs.

Deserts: Because of the deep penetration of P-band radiation into arid soils, the dominant scattering in some 
desert environments is likely to be from the underlying bedrock, unless there is significant attenuation due to the presence of mineral salts or iron-rich materials. If there is no overlying vegetation and no change in the soil moisture, the backscattering coefficient is then expected to be very stable. The Selima Sand Sheet in the eastern Sahara, which is flat, extensive, hyper-arid and has homogeneous sandstone bedrock covered by aeolian sediments less than $1 \mathrm{~m}$ thick, is a strong potential reference target. However, a key question is whether it will provide sufficient SNR at P-band. Based on airborne measurements in Tunisia, L-band measurements from ALOS-1 and -2 and modeling, the co-polar backscattering coefficient may be around $-23 \mathrm{~dB}$, while the cross-polarized value will be significantly smaller. This is insufficient, given that the target NESZ for BIOMASS is -27 $\mathrm{dB}$. There is, however, little chance of measuring the actual backscattering coefficients before launch, as the Selima Sand Sheet lies across the boundary of southern Egypt and Northern Sudan, and this is too dangerous a region for airborne or in situ campaigns. Our first measurements will therefore be acquired during the $\mathrm{CP}$.

Icesheets: Because of the deep penetration of P-band radiation into the ice in the dry zone where melt never occurs we expect its backscattering coefficient to be very stable. SOTR restrictions will prevent measurements in the Arctic, so the issue is whether there are sufficiently bright, extensive, stable, homogeneous regions in the Antarctic. Since there are no available P-band measurements, L-band data provide the best means of investigating this. In particular, Aquarius L-band scatterometer measurements with $29^{\circ}$ incidence angle from the Dome-C region are consistently bright and stable throughout the year (note that the incidence angle of BIOMASS varies from $23^{\circ}$ in near range to $33.75^{\circ}$ in far range). However, we have no reliable means to estimate the P-band backscattering coefficient, so without an airborne campaign our first chance to characterize properly the radiometry of Dome-C at P-band will be during the $\mathrm{CP}$.

Permanent Point Scatterers (PPS): Because there are so few multi-temporal P-band datasets, it has only been possible to assess the value of PPSs in BIOMASS calibration using PALSAR data. From this analysis [3], the most useful types of target appear to be desert with isolated rocks, savanna with isolated trees and urban areas. Radiotelescope antennas are unlikely to be useful as calibrators unless they are pointed towards the satellite. Far fewer suitable targets are found in the cross-polarized channels than in the co-polarized channels, which will hinder or even prevent calibration of cross-polarized channel quantities. A further caveat is that scaling of RCS and clutter to the BIOMASS wavelength and resolution is likely to reduce the number of PPSs of sufficient quality to be used in calibration. Hence, as in the case of deserts and icesheets, identification and characterization of suitable PPSs will be a crucial element of the $\mathrm{CP}$.
Common issues: Common concerns for all three types of target of opportunity are ionospheric and incidence angle effects. Faraday rotation (FR) would seriously affect radiometry but, as noted in Section 2, it can be readily corrected to an accuracy of better than $1^{\circ}$, whereupon the associated radiometric distortion is negligible. Potentially damaging for the use of icesheets in calibration is high latitude ionospheric scintillation, which can affect radiometry through spatial shifts, spectral shifts/broadening and increased azimuth ambiguities. However, current calculations suggest that these effects will have negligible radiometric consequences.

Potentially more serious is that the change in backscatter with incidence angle is unknown for all three types of target, but such knowledge is needed if they are to be used to correct elevation antenna pattern effects. In the absence of further campaign data, the best chance to obtain this information is during the $\mathrm{CP}$, and this will form part of the target identification and characterization during this phase.

\section{POLARIMETRIC CALIBRATION}

The general calibration problem relating the measured (M) and true $(\mathbf{S})$ scattering matrices is given by

$$
\begin{array}{r}
{\left[\begin{array}{ll}
M_{h h} & M_{v h} \\
M_{h v} & M_{v v}
\end{array}\right]=A(r, \theta) e^{j \varphi}\left[\begin{array}{cc}
1 & \delta_{2} \\
\delta_{1} & f_{1}
\end{array}\right] \times\left[\begin{array}{cc}
\cos \Omega & \sin \Omega \\
-\sin \Omega & \cos \Omega
\end{array}\right] \times\left[\begin{array}{ll}
S_{h h} & S_{v h} \\
S_{h v} & S_{v v}
\end{array}\right]} \\
\times\left[\begin{array}{cc}
\cos \Omega & \sin \Omega \\
-\sin \Omega & \cos \Omega
\end{array}\right] \times\left[\begin{array}{cc}
1 & \delta_{3} \\
\delta_{4} & f_{2}
\end{array}\right]+\left[\begin{array}{cc}
N_{h h} & N_{v h} \\
N_{h v} & N_{v v}
\end{array}\right]
\end{array}
$$

Absolute amplitude and phase calibration consists of estimating and compensating for the $A(r, \theta) e^{j \varphi}$ in the presence of noise $\left(N_{p q}\right)$, while polarimetric calibration involves correcting for non-zero values of cross-talk $\left(\delta_{i}\right)$ and non-unity values of channel imbalance $\left(f_{i}\right)$ in the presence of FR with angle $\Omega$. System requirements are $\left|\delta_{i}\right|<$ $-30 \mathrm{~dB}$ and $\left|f_{i}-1\right|<-34 \mathrm{~dB}[2]$ and the system is intended to provide this without calibration. However, methods are needed to be verify that these conditions are met; if not, corrections will be required.

\subsection{Estimating system errors using a transponder}

Using a transponder or specially designed passive polarimetric calibrators, it is possible to provide 4 independent scatterers in which only one of the elements of the true scattering matrix is non-zero. Using (11), this leads to an over-determined set of equations for the system errors, $\Omega$ and system noise, which also allows for imperfections in the calibrators [3]. The solution scheme derived in [5] led to the conclusion that extremely accurate estimates of $\Omega$ were needed to give adequate estimates of system errors, and this required the calibration devices to be located almost exactly 
where $\Omega=0$ (near the magnetic equator) or provision of a separate estimate of $\Omega$. However, later work showed that solving the system of equations using non-linear optimization removed this restriction and in fact indicated that locating the devices at mid-latitudes conveyed several advantages [1]. Current work aims to consolidate these findings and to quantify the Signal to Clutter Ratio needed for system errors are to be measured to sufficient accuracy to test if the system is operating within specification.

\subsection{Estimating system errors using distributed targets}

Because the transponder(s) will be visited so infrequently, routine verification of polarimetric fidelity must use the properties of targets of opportunity. There are already welldeveloped methods to estimate cross-talk using distributed targets if the following properties are met [6]:

1. reflection symmetry, so that the co- and cross-polarized returns are uncorrelated;

2. backscattering coefficient large enough to dominate noise;

3. negligible Faraday rotation.

Such methods should be applicable when BIOMASS is in orbit over forest regions in flat areas near the magnetic equator. In fact, because of the geometry of the magnetic equator, such conditions are met only in Amazonia.

A crucial requirement to apply these methods is location of the true zero-FR line, but this is hindered by the ambiguity between small angles of FR and the phases of the cross-talk terms in (11) (the same values of the scattering matrix would be measured in either case). This problem has been solved, however, by using the fact that the FR is inversely proportion to the square of radio frequency. This means that FR varies linearly with frequency across the chirp signal used by BIOMASS, and there should be sufficient sensitivity to measure this variation. An accurate estimate of the true FR, $\Omega_{0}$, is then given by

$$
\frac{d \hat{\Omega}}{d f} \approx-2 \frac{\Omega_{0}}{f_{0}}
$$

where $\hat{\Omega}$ is the estimated FR as a function of frequency, $f$, and $f_{0}$ is the centre frequency of the chirp $(435 \mathrm{MHz}$ for BIOMASS) [1]. This estimate is almost unaffected by crosstalk (however, the linearity of the FR-dependence on frequency assumes that cross-talk is the same across all frequencies, and measurements on PALSAR data suggest this may not always be true [1]).

\section{GEOLOCATION}

BIOMASS images must be geolocated to an accuracy of 25 $\mathrm{m}$ [4], which corresponds roughly to half the resolution of the 6-look (in azimuth) ground range BIOMASS product. A problem in meeting this requirement is the ionospheric range delay, $r_{s d}$ (7b). However, we can use measurements of FR and the relation between FR and $r_{s d}(8)$ to estimate the delay, with an accuracy controlled by the variance of the estimator (5). This can then be used to correct the geometric distortion in the range direction. However, this method breaks down near the magnetic equator because of the dependence of FR on the angle between the propagation direction and the magnetic field (8). This defines a region straddling the zeroFR line where the required accuracy cannot be met. Calculations of this zone for a centred dipole magnetic field model are given for BIOMASS parameters in Table 1, using the notation in (5) and assuming ionospheric delay is the only relevant term in the geolocation error budget. Since $1^{\circ}$ in latitude corresponds roughly to $110 \mathrm{~km}$, it can be seen that in the best case (SNR $=20 \mathrm{~dB}, L=10000)$ the excluded zone is only around $10 \mathrm{~km}$ in width. The BIOMASS singlelook resolution is $25 \mathrm{~m}$ in slant range and $8.3 \mathrm{~m}$ in azimuth, so after 6-look averaging in azimuth the ground pixel size is roughly $50 \mathrm{~m} \times 50 \mathrm{~m}$. Hence the best-case averaging window corresponds to $2 \mathrm{~km} \times 2 \mathrm{~km}$ on the ground. The TEC is likely to be almost constant over the associated window at ionospheric heights, so it is reasonable to use such window sizes, or even larger ones. It is more complicated to perform similar calculations in the azimuth direction, for which TEC gradients cause shifts, but [1] reports similar findings.

Table 1: The range of latitudes over which the $25 \mathrm{~m}$ BIOMASS geolocation accuracy in range cannot be met using FR measurements

\begin{tabular}{|c|c|c|}
\hline$L$ & 100 & 10000 \\
\hline $\mathrm{SNR}=10 \mathrm{~dB}$ & $0.28^{\circ}$ to $3.44^{\circ} \mathrm{N}$ & $1.70^{\circ}$ to $2.02^{\circ} \mathrm{N}$ \\
\hline $\mathrm{SNR}=20 \mathrm{~dB}$ & $1.35^{\circ}$ to $2.37^{\circ} \mathrm{N}$ & $1.81^{\circ}$ to $1.91^{\circ} \mathrm{N}$ \\
\hline
\end{tabular}

\section{REFERENCES}

[1] Quegan, S., M. Lomas, K. P. Papathanassiou, J-S. Kim, S. Tebaldini, D. Giudici, M. Scagliola, P. Guccione, J. Dall P. Dubois-Fernandez and P. Paillou, BIOMASS External Calibration Study, Final Report, ESA/ESTEC Contract No. 4000116784/15/NL/CT, 2016.

[2] N. C.Rogers, and S.Quegan, "The accuracy of Faraday rotation estimation in satellite Synthetic Aperture Radar images", IEEE Trans. Geosci. Remote Sensing, 52, no. 8, pp. 4799 - 4807, 2014.

[3] P. Guccione, M. Scagliola and D. Giudici, "Low-frequency SAR radiometric calibration and antenna pattern estimation by using stable point targets," IEEE Trans Geosci. Remote Sensing, vol. PP, no. 99, pp. 1-12, doi: 10.1109/TGRS.2017.2752228.

[4] ESA, Biomass System Requirement Document for Phase $B 2 / C / D / E 1, \quad B I O-R S-E S A-S Y-0011$, Issue 1, Revision 1, $10 / 03 / 2016$.

[5] J. Chen, S. Quegan \& X. J. Yin, "Calibration of spaceborne linearly polarized low frequency SAR using polarimetric selective radar calibrators," Progress in Electromagnetic Research, 114, pp. 89-111, 2011.

[6] S Quegan, "A unified algorithm for phase and cross-talk calibration of polarimetric data - theory and observations", IEEE Trans Geosci. Remote Sensing, 32, pp. 89-99, 1994. 